\title{
AN ANALYSIS OF DRIVING PERFORMANCE MEASURES USED TO ASSESS THE EFFECTS OF MEDICATIONS ON DROWSINESS, SEDATION, AND DRIVING IMPAIRMENT
}

\author{
Ginger S. Watson, Ph.D. \\ Director, Human Factors Laboratory \& Senior Research Scientist \\ National Advanced Driving Simulator \\ E-mail: g-watson@uiowa.edu \\ John M. Weiler, M.D. \\ Professor, Department of Internal Medicine \\ College of Medicine \\ E-mail: john-weiler@uiowa.edu \\ Julie C. Qidwai, B.S. \\ Staff Research Assistant \\ National Advanced Driving Simulator \\ E-mail: julieq@nads-sc.uiowa.edu \\ George G. Woodworth, Ph.D. \\ Professor, Statistics \& Actuarial Science \\ College of Liberal Arts \\ E-mail: george-woodworth@uiowa.edu \\ Susan A. Quinn, R.N., B.S.N., CCRC \\ Clinical Research Coordinator \\ Department of Internal Medicine \\ E-mail: susan-quinn@uiowa.edu
}

The University of Iowa

Iowa City, IA USA

Summary: The objective of this paper was to discuss driving scenarios and associated driving performance measures on their ability to demonstrate drowsiness, sedation, and driving impairment. The basis of this paper was a study that utilized a randomized, double-blind, double-dummy, four-treatment, four-period crossover trial in the Iowa Driving Simulator (IDS). Participants were 40 licensed drivers with seasonal allergic rhinitis who were 25 to 44 years of age. Treatments were Fexofenadine, diphenhydramine, alcohol, or placebo, given at weekly intervals before participants drove for 1 hour in the IDS. Measures examined included coherence, amplitude, phase angle, RMS error, following distance and behavior, lane keeping, response to unexpected vehicle intrusion and drowsiness.

Study results show that sedating antihistamines impair driving performance as seriously as alcohol. Statistically significant but small correlations were found between subjective drowsiness and minimum following distance, steering instability, and left-lane excursions but no correlation was greater than 0.21 . Drowsiness was a weak predictor of driving impairment. This paper discusses these and other finding with an emphasis on the adequacy of driving scenarios and the sensitivity of driving performance measures analyzed. 